\title{
Kapitel 8 \\ Das Deutsche: auf dem Weg zu einem Sprachporträt
}

\section{Einstieg}

In diesem abschließenden Kapitel nähere ich mich, ähnlich wie in Kapitel 2, meinem Gegenstand mithilfe mehrerer Neuansätze bzw. aufeinander aufbauender Runden an. Der Weg zu einem Sprachporträt des Deutschen wird dabei in der ersten Runde darin bestehen, die Ergebnisse der vorangehenden Kapitel zusammenzufassen - immer unter der Perspektive, welche charakteristischen Merkmale dieser Sprache dabei jeweils zu Tage getreten sind. In der zweiten Runde setze ich diese Merkmale in Beziehung zu den Aussagen, die in der typologischen und sprachvergleichenden Sprachwissenschaft zu Eigenschaften des Deutschen gemacht wurden. Dies betrifft zum einen die bekannten Kriterien der morphologischen Typologie (wie etwa den Grad an Synthese und Fusion in der Flexion), zum anderen auch die Syntax. Dabei gehe ich auch auf die Frage ein, was überhaupt die Verschiedenheit des menschlichen Sprachbaus ermöglicht und vorangetrieben hat. Ich setze mich mit der heute aktuellen Idee auseinander, ein Wettbewerb unterschiedlicher Motivationen sei hier die treibende Kraft. In der dritten Runde schließlich beziehe ich mich auf arealtypologische Überlegungen, frage also, ob und inwiefern das Deutsche ein Vertreter des SAE, des „Standard Average European“, ist.

Das Unternehmen ist, was die zweite und dritte Runde betrifft, riskant. Auch in Bezug auf Sprachen besteht bei der Typisierung die Gefahr der Übergeneralisierung. Pauschale Einordnungen werden oft der Vielfalt feiner Differenzierungen und den Divergenzen innerhalb des Sprachsystems nicht gerecht. Wenn z. B. die Flexionsmorphologie des Verbs einem bestimmten, z. B. dem fusionierenden Typ zuzuordnen ist, muss die der nominalen Kategorien nicht notwendigerweise demselben Typ angehören. Übergreifende Harmonie kann nicht vorausgesetzt werden. Dabei steht im Hintergrund immer die Frage nach der Systemhaftigkeit einer Sprache als einer historisch gewordenen Institution. 


\title{
2 Erste Runde: Welche Charakteristika des Deutschen wurden vorgefunden?
}

\section{Kapitel 2}

\begin{abstract}
Hier werden die Grundlagen einer funktionalen Sicht auf Wortschatz und Grammatik herausgearbeitet: von einer Klärung des Verhältnisses dieser beiden Ebenen über eine Annäherung an das Geheimnis von Wortbedeutungen und an den Sinn grammatischer Regeln bis zum sprachlichen Handeln und dem Konstrukt der Proposition mit den Bausteinen Referenz und Prädikation.
\end{abstract}

Was die beiden großen Teilbereiche angeht, die uns hier beschäftigen, Wortschatz und Grammatik, so unterscheidet sich das Deutsche nicht von anderen europäischen Sprachen. Es liegt folgende grobe Arbeitsteilung vor: Der Wortschatz stellt mit seinem Inventar an bedeutungstragenden Einheiten Instrumente des Klassifizierens und Kategorisierens bereit, aber auch Mittel der sozialen, evaluativen und emotiven Bewertung. Die Grammatik enthält die Spielregeln für den Umgang mit den signifikativen Einheiten. Wortbedeutungen sind keine fertigen mentalen Einheiten. Auch die grammatischen Regeln sind sprachspezifisch, ihr Sinn aber liegt außerhalb: Die mit ihrer Hilfe erzeugten kommunikativen Einheiten dienen dazu, die Außen- wie unsere Innenwelt zu erschließen, den zwischenmenschlichen Verkehr zu regeln, Austausch und Kooperation zu strukturieren; die Regeln der Grammatik stellen also Formen des Handelns für unseren Gebrauch zur Verfügung. Die grundlegenden Typen des sprachlichen Handels - nach Searle repräsentative, kommissive, direktive, expressive und deklarative - dürften universale Geltung haben. Jede dieser Klassen steht für zahlreiche unterschiedliche sprachliche Handlungen, die vor allem durch Sprechaktverben benannt oder auch in explizit performativer Manier mit ihrer Hilfe direkt vollzogen werden können. Auf der grammatischen Ebene sind die Mittel äußerst beschränkt. Im Deutschen werden die Satzarten Aussagesatz, (Entscheidungs-)Fragesatz und Aufforderungssatz in erster Linie durch die Verbstellung differenziert; hinzu kommen können z. B. der Verbmodus sowie intonatorische Merkmale. Daraus ergibt sich, dass Sätze ihrer Form nach nur über ein illokutives Potenzial verfügen, das in der konkreten Verwendung auf der Basis der Satzbedeutung über Schlussfolgerungen mehr oder weniger vereindeutigt wird.

Neben der illokutiven Kraft ist die Proposition, der Sachverhaltsentwurf, konstitutiv für Satzbedeutungen. Deren Bausteine, Referenz und Prädikation also die Spezifikation von Gegenständen der Rede und die Zuschreibung von Eigenschaften an diese Gegenstände bzw. von Relationen zwischen ihnen sind möglicherweise in allen Sprachen „anwesend“. Aber nicht unbedingt in dem uns vertrauten Modus, wo beide auch syntaktisch relativ klar geschieden 
sind. In den europäischen Sprachen stehen in erster Linie Nominalphrasen für die Referenz und Verben oder auch Verbalphrasen für die Prädikation in der Weise, dass das verbale Prädikat Leerstellen für seine Mitspieler, die Argumente, eröffnet. Diese bezeichnen in der Regel die Gegenstände, auf die sich die Zuschreibungen des Prädikats beziehen. In den europäischen Sprachen ist zudem eine der Stellen, die des Subjekts, gegenüber den anderen semantisch durch Prominenz und morphologisch durch Kongruenz mit dem Prädikatsverb besonders ausgezeichnet.

Referenz kann nicht einfach mit der Identifikation von existenten Gegenständen der realen Welt gleichgesetzt werden; auch über nur denkbare Objekte und in unterschiedlicher Weise konstruierte Kollektionen prädizieren wir, und insofern sind sie in einem weiteren Sinne Gegenstände unserer Referenz. Neben dem gestischen und dem verbalen „Zeigen“ mittels deiktischer Ausdrücke können Eigennamen zwar als das einfachste Mittel der Referenz erscheinen. Zumindest unter Gedächtnisgesichtspunkten sind sie aber nicht ökonomisch und nur Gegenständen vorbehalten, die uns Menschen besonders nahe und wichtig sind.

\section{Kapitel 3}

Folgende Schwerpunkte sind hier gesetzt: Das Verb ist als Prädikatsausdruck im Rahmen der für europäische Sprachen typischen Satzstruktur (vgl. Kapitel 2) der Kern des Sachverhaltsentwurfs. Darauf sind seine Kategorisierungen abgestimmt, Mit dem Tempus werden Vorgaben für dessen zeitliche Einordnung gemacht, mit dem Modus für dessen Realitätsbezug. Durch Person und Numerus wird die Kongruenz mit dem Subjekt (im Ungarischen partiell auch dem direkten Objekt), und damit die Anbindung des oder der zentralen Mitspieler des Prädikats gesichert. Das Genus Verbi ermöglicht mit Aktiv und Passiv unterschiedliche Perspektiven auf den Sachverhalt. Es ist somit eines der grammatischen Mittel, mit denen Sprecher ihre Sicht auf Geschehnisse umsetzen können. Das Verb strukturiert nicht zuletzt durch seinen Valenzrahmen sowohl den semantischen Beitrag seiner Komplemente als auch deren syntaktische Realisierung vor.

In den europäischen Sprachen sind in der Regel mindestens drei Tempora als grammatische Kategorien des Verbs - gegeben; ein solcher grammatischer Ausdruck des Zeitbezugs ist aber nicht in allen Sprachen der Welt vorhanden. Im Standarddeutschen werden sechs Tempora unterschieden: Nur zwei, Präsens und Präteritum, werden durch einfache Verbformen kodiert, Futur (wie in anderen germanischen Sprachen) und die Perfekttempora (wie in germanischen und romanischen Sprachen) durch periphrastische (zusammengesetzte) Formen. Der ähnlichen formalen Struktur der Tempora etwa im Deutschen und Englischen stehen aber gravierende Unterschiede in deren Gebrauch gegenüber. Die Alternative in der Wahl des Perfekthilfsverbs (haben versus sein) teilt das Deutsche hingegen mit dem Niederländischen und Dänischen, aber 
auch mit einer Reihe von romanischen Sprachen. Die Kategorie Aspekt haben im Vollbild nur das Polnische und andere slawische Sprachen. Nur partiell, aber in unterschiedlicher Weise, ist der Aspekt im Englischen und Französischen grammatikalisiert, im Deutschen gar nicht - sieht man vom am-Progressiv einmal ab. Die aus dem Germanischen ererbte Unterscheidung zwischen starken und schwachen Verben, die markante Unterschiede bei Tempus- und Modusbildung mit sich bringt, ist im Deutschen, etwa im Vergleich zu den vereinfachenden Tendenzen im Englischen, noch mit einer Vielfalt von Mustern erhalten, deren Systematik allerdings nicht leicht zu durchschauen ist.

Das Deutsche verfügt über zwei nach Tempora ausdifferenzierte Konjunktivgruppen: den formal auf dem Indikativ Präsens fußenden Konjunktiv 1 und den auf dem Indikativ Präteritum fußenden Konjunktiv 2. Konjunktiv 1 ist in erster Linie eine Form für die indirekte Redewiedergabe, Konjunktiv 2 eine zum Ausdruck von Potenzialität und Irrealität. Beide signalisieren ein Abrücken vom Normalfall des Weltbezugs und der sprecherseitigen Gültigkeitsgarantie. Die auf Basis des Präsens und Präteritum gebildeten synthetischen Konjunktive sind nicht hinreichend differenziert vom Normalmodus Indikativ, es kommt zum Zusammenfall an einzelnen Paradigmenstellen oder gar wie beim Konjunktiv 2 der schwachen Verben zum vollständigen Synkretismus. Als Reparaturverfahren springt einerseits der Konjunktiv 2 für den Konjunktiv 1 ein, zum anderen tritt die analytische würdeForm an die Stelle des Konjunktivs 2. Die markierten Modi anderer europäischer Sprachen, etwa der Subjonctif des Französischen, sind keine Indirektheitsmodi, sondern signalisieren - in der Regel in Nebensätzen - die Erwünschtheit oder allgemeiner die Offenheit des dargestellten Sachverhalts.

Mithilfe der lexikalischen Bedeutung des Prädikatsverb legen wir offen, wie wir Sachverhalte sehen, oder besser gesagt: inszenieren - wobei wir uns, wenn wir wahrhaftig sein wollen, an das halten, was „Sache“ ist. Die Gegebenheiten lassen aber große Spielräume zu, z. B. im Zuschnitt von Ereignissen und deren Auffassung etwa als von Menschen verantwortete Handlung oder als selbstlaufender Prozess. Gewisse Präferenzen in der Modellierung von Ereignissen - z. B. Fokussierung von Bewegungsabläufen versus Zielen der Fortbewegung - sind sprachspezifisch angelegt, was aber nicht unbedingt als Unterschiede in den „Weltbildern“ interpretiert werden muss. Grammatische Mittel der Inszenierung von Ereignissen sind unter anderem Passivsetzung und Reflexivierung. Das Deutsche verfügt über ein vergleichsweise breites Spektrum an Passivformen. Ebenso kann, ähnlich wie etwa im Französischen und Polnischen, die Repräsentation selbstlaufender Prozesse und vor allem von Körperbewegungen und Emotionen mithilfe von Reflexivkonstruktionen aus transitiven Verben, also in der Regel fremdgerichteten Prädikationen, abgeleitet werden. 
Die Komplemente des Prädikatsverbs müssen formal voneinander unterscheidbar sein. Von den formalen Möglichkeiten der Unterscheidung durch Kasus, Präposition und lineare Ordnung im Satz werden im Deutschen die beiden erstgenannten genutzt, während sich etwa Englisch z. T. der Linearität bedient. Das Deutsche hat sich, wenn auch nicht immer deutlich sichtbar, vier Kasus bewahrt gegenüber um die zwanzig Kasus im Ungarischen, sieben im Polnischen sowie - nur bei gewissen Pronomina - drei im Französischen und nur zwei im Englischen. Schon aus dieser zahlenmäßigen Differenz ergibt sich, dass es zwischen den Kasus bzw. den durch sie oder andere Mittel realisierten syntaktischen Funktionen und semantischen Rollen keine parallelen Schnitte geben kann. Der deutsche Subjektsnominativ ist offen für alle semantischen Rollen, der Akkusativ des direkten Objekts hat niemals die Agens-Rolle inne, der Dativ weder die des Agens noch die des Patiens. In den anderen Vergleichssprachen dürfte für den Subjektsnominativ das Gleiche gelten, da aber z. B. im Englischen kein Dativ vorhanden ist, reicht der Akkusativ auch in die Domäne des (dativischen) indirekten Objekts des Deutschen hinein.

\section{Kapitel 4}

Zum einen werden hier Nominalphrasen, die in erster Linie referenzielle Ausdrücke sind, in ihrem syntaktischen Aufbau beschrieben. Weitere Gesichtspunkte sind die Wortartenunterscheidung im nominalen Bereich, die nominalen Kategorisierungen Kasus, Genus und Numerus, die grammatische Gliederung des Substantivwortschatzes sowie ein exemplarischer Fall der Attribution, die Possessivattribute innerhalb der Nominalphrase.

In der Syntax des nominalen Bereichs verkörpert sich der hierarchische Aufbau von Wortgruppen bzw. Phrasen in exemplarischer Weise: Attribute modifizieren den meist substantivischen Kopf der Nominalphrase; Determinative, insbesondere Artikel, können hinzutreten. Bei Attribuierung werden im Deutschen Vorund Nachbereich des Kopfs genutzt, im Ungarischen praktisch nur der Vorbereich; im Englischen, Französischen und teilweise auch Polnischen erscheinen im Gegensatz zum Deutschen Adjektive und Partizipialphrasen meist nach dem Kopf.

Die Debatte um die universale Geltung von Wortartunterscheidungen ist noch nicht entschieden. Im Deutschen wie auch anderen europäischen Genussprachen (Polnisch, Französisch) stechen die Substantive, die Kategorisierungen wie Kasus, Genus und Numerus mit anderen nominalen Wortarten teilen, mit ihrem konstanten Genus klar hervor. Die Substantivgroßschreibung ist ein besonderes Charakteristikum des deutschen Schriftsystems. Die mögliche Divergenz zwischen der syntaktischen Funktion als Kopf der Nominalphrase und lexikalischer Wortartenzugehörigkeit - Adjektive und andere Elemente können 
„nominalisiert“ werden, Substantive können „desubstantiviert“ werden - führt zu Problemen, in denen sich auch die Dynamik der Sprachentwicklung widerspiegelt. Die Wortart Adjektiv ist weniger wohl bestimmt. Neben semantisch und syntaktisch prototypischen ,Eigenschaftswörtern' gibt es auch Teilklassen, etwa die ,Relationsadjektive“ mit z. T. abweichendem morphosyntaktischem Verhalten. Für das Deutsche ist im Unterschied zu den Kontrastsprachen bemerkenswert, dass die Komparation rein flexivisch, nicht analytisch geschieht, und dass die Modifikation von Substantiv und Verb sich gleichermaßen des Adjektivs bedient - nicht etwa einer adverbialen Ableitung aus dem Adjektiv. Ebenfalls zu den nominalen Wortklassen gehören die Pronomina und Determinative: Hier ist die zwischensprachliche Divergenz besonders groß. Besonderheit des Deutschen ist, dass selbstständige Pronomina teilweise von ihren determinativischen Gegenstücken flexionsmorphologisch geschieden sind - wie etwa bei kein Mensch versus keiner.

Die nominalen Kategorisierungen Kasus, Genus und Numerus haben ganz unterschiedliche Funktionen. Numerus, also nominale Quantifikation in der binären Version Singular versus Plural, weisen die europäischen Sprachen generell auf, Kasus als Mittel der syntaktischen Relationierung in abgestuftem Maße, das Genus als Sortierungsverfahren für den Wortschatz und - indirekt - für die Gegenstände der Welt fehlt z. B. im Ungarischen, Finnischen oder Türkischen ganz. Die nominalen Wortarten des Deutschen flektieren nach Kasus und Numerus, außer den Substantiven auch nach Genus. Am differenziertesten ist dabei die pronominale Flexion. Die Kasusunterscheidung ist beim Substantiv wenig ausgeprägt; die Numerusunterscheidung, bei der mehrere Flexionstypen zu beobachten sind, ist dagegen hervorgehoben. Insgesamt herrscht in der Nominalphrase das Prinzip der flexivischen Kooperation, bei der Artikel bzw. Determinative die Hauptlast der Kasusunterscheidung tragen, aber auch ein attributives Adjektiv oder gar der substantivische Kopf gegebenenfalls ihren Teil beitragen. Genus und Sexus bilden in den Sprachen der Welt eine häufig anzutreffende „natürliche“ Allianz. In indoeuropäischen Sprachen besteht Asymmetrie zwischen den Genera, die zugunsten des Maskulinums und zu Lasten des Femininums geht, mit Folgen für die Sichtbarkeit des weiblichen Sexus. Daraus ergibt sich nicht nur im Deutschen ein herausragendes Feld der Sprachentwicklung.

Weniger auffällig als die klassischen nominalen Kategorien ist der Nominalaspekt, mit dem unterschiedliche Seinsarten wie Propria, Individuativa und Kontinuativa differenziert werden. In einer Artikelsprache wie dem Deutschen sind die Unterschiede relativ klar grammatikalisiert. Die universal gültigen Nominalhierarchien, vor allem die Belebtheitshierarchie, schlagen sich im Deutschen etwa in der besonderen grammatischen Aktivität der Personalpronomina (z. B. im Hinblick auf Kasusdifferenzierung) deutlich nieder. 
Die kognitiv zentrale und auch in verbalen Prädikationen vertretene Domäne der Possession ist in Form der Possessivattribute semantisch weit über den Kern des materiellen Besitzes oder der unveräußerlichen Zugehörigkeit bei Verwandtschaft und Körperteil ausgedehnt. Im Deutschen sind Possessivattribute (Genitiv und von-Phrasen) wie in anderen europäischen Sprachen als die prototypische Form der Attribution zu betrachten. Das Nebeneinander der beiden Formen der Markierung durch Kasus und Präposition am Attribut, also am ,Dependens', verweist auf Gemeinsamkeiten mit anderen germanischen Sprachen; im Detail bestehen aber etwa zum Englischen wieder deutliche Gebrauchsunterschiede. Das Possessivpronomen des Deutschen ist mit dem Reflex von Genus- und Numerusunterscheidungen des Bezugsausdrucks durch seinen Stamm (seinversus $\left.i h r^{-}\right)$kognitiv vergleichsweise komplex; auch hier zeigen sich Tendenzen zur Vereinfachung.

\section{Kapitel 5}

Thematisiert wird die Grammatik der Supplemente, der valenzfreien Zugaben zum Propositionsausdruck. Vor allem aber geht es um die lineare Ordnung im Satz. Daneben werden auch die Lesarten von Satz als Einheit der Grammatik und der Kommunikation geklärt.

Supplemente sind semantisch und syntaktisch „systemfreier“ als Komplemente. Sie erfüllen unseren Wunsch nach der Ergänzung des notwendigerweise zu Sagenden um alles, was zu sagen umständehalber nützlich bzw. sozial oder argumentativ angemessen ist. Situative, konditional-kausale und andere Supplemente gibt es sprachübergreifend z. B. in Form von Adpositionalphrasen, Adverbien, aber auch Nebensätzen. Dennoch prägt das jeweilige System die Art ihres Ausdrucks. Bei Adpositionalphrasen folgt besonders die Umfunktionalisierung lokal-temporaler Prä- oder Postpositionen für abstraktere Zwecke wie die Kausalität z. T. allgemeineren konzeptuellen Mustern, die Wahl spezifischer Ausdrücke (wie im Deutschen bei aus Angst, vor Vergnügen, über das Geschenk) ist sprachspezifisch. Eine Merkwürdigkeit des Deutschen stellen die Abtönungspartikeln dar, die in anderen europäischen Sprachen nur rudimentär ausgebildet sind. Auch der Gebrauch von sekundären, insbesondere resultativen Prädikativen in Form von Adjektiven ist ein Charakteristikum des Deutschen.

Von besonderem Interesse ist in Sprachvergleich und Typologie die Linearität des Satzes. Sie übersetzt die hierarchische Ordnung - was zusammengehört, was Teil wovon ist - in ein Nacheinander. Diese Eindimensionalität ist Ursache für zahlreiche Ambiguitäten, die nicht immer durch andere sprachliche Mittel aufgelöst werden können. Das Deutsche findet besondere Aufmerksamkeit, weil 
es sich nicht so leicht in die einfachen Muster wie SOV, SVO einordnen lässt. So ist auch umstritten, ob das Deutsche eine Grundordnung hat und wenn ja welche. Bei näherer Betrachtung ist die Feldertopologie des Deutschen herausstechend. Insgesamt herrscht in der Wortstellung - oder besser: Konstituentenstellung - eine Mischung aus Freiheit und Ordnung: Das Vorfeld muss nicht durch den grammatisch designierten Vertreter, das Subjekt, besetzt sein, im Mittelfeld gibt es schon rein systematisch miteinander konkurrierende Prinzipien, darüber hinaus noch kommunikativ motivierte Umordnungen, das Nachfeld wirkt ohnehin leicht chaotisch. Hier zeigen sich klare Abweichungen von den Kontrastsprachen mit ihrer stärker ausgeprägten Subjekt-zuerst-Strategie (Englisch, Französisch) oder gar der Topikprominenz (Ungarisch). Am ähnlichsten, wenn auch ohne Klammerstruktur, erscheint Polnisch, das sich noch mehr Freiheiten erlaubt. Immerhin orientieren sich die betrachteten Sprachen grundsätzlich am Prinzip Alt vor Neu, Bekanntes vor Unbekanntem. Unterschiede betreffen die Art, inwieweit das in feste syntaktische Formen gegossen wird.

Sätze können als Komplemente oder Supplemente oder auch als gleichberechtigte Glieder Teile eines anderen Satzes, eines Ganzsatzes, sein. Neben dem Satz gibt es knappere Formen von Mitteilungseinheiten, die Illokution und Proposition ohne Beiwerk, auf das in der jeweiligen Situation verzichtet werden kann, zum Ausdruck bringen.

\title{
Kapitel 6
}

\begin{abstract}
Am Text bzw. der Textualität interessieren aus grammatischer und vergleichender Perspektive folgende Themen: die Kohäsionsmittel, also was den Text formal zusammenhält - hierher gehören die Mittel des Rückbezugs und der inhaltlichen Konnexion sowie die Temporaund die möglichen Einflüsse der textuellen (oder auch situativen) Umgebung auf die Struktur von Mitteilungseinheiten, insbesondere die Möglichkeiten der elliptischen Reduktion.
\end{abstract}

Der grammatische Beitrag zur Textualität ist vergleichsweise gering. Allerdings kann die formale Kohäsion der inhaltlichen Kohärenz von Texten dienlich sein. Mittel der Kohäsion sind Referenzausdrücke, die die Satzgrenze überschreiten helfen, in erster Linie die anaphorischen Pronomina, sowie die Verknüpfung durch spezielle Konnexionsmittel. Bei der referenziellen Textkohäsion liegt die entscheidende Weichenstellung zwischen den ,Pro-Drop'-Sprachen und den Sprachen, bei denen Personalpronomina obligatorisch sind. Das Deutsche gehört wie die anderen germanischen Sprachen zu den Nicht-Pro-Drop-Systemen, während die romanischen Sprachen (mit Ausnahme des Französischen) und die slawischen wie auch das Ungarische in unterschiedlichem Maße ProDrop favorisieren. Die Genus-Distinktion bei den Personalia ist ein vergleichs- 
weise probates Mittel des Deutschen für die Klärung, wie ein Pronomen zu verstehen ist, die ,Anaphernresolution'.

Die Konnektoren können im Deutschen an unterschiedlichen Positionen im Satz auftreten, viele auch bevorzugt im Vorfeld - Vergleichbares ist etwa im Englischen oder Französischen aufgrund der Subjekt-zuerst-Regel kaum möglich. Eine Besonderheit des Deutschen sind zweiteilige Konnektoren, die (wie bei deswegen, darauf oder worauf) aus einem Verweisausdruck und einer Präposition bestehen.

Die Tempora können verschiedene Formen der zeitlichen Zugänglichkeit zwischen einzelnen im Text geschilderten Ereignissen wie Überlappung oder zeitliche Sequenz signalisieren, sind aber viel zu unspezifisch, um hier klare Verhältnisse zu schaffen. Daneben haben sie textsortenbezogene wie textstrukturierende Funktion. Die Reliefbildung, die etwa im Französischen durch den Aspekt bewirkt werden soll, ist im Deutschen kaum möglich.

Charakteristisch für elliptisches Schreiben oder Sprechen ist im Unterschied zu den in Kapitel 5 erörterten illokutiv und propositional vollständigen Kurzformen die Unvollständigkeit der verbalisierten Proposition. Der Text, der Diskurs oder die Situation müssen Referenten oder Prädikate liefern bzw. erschließen lassen. Verbalisiert wird jeweils nur das, was im Fokus des Interesses steht.

\section{Kapitel 7}

Hier befassen wir uns zunächst mit folgenden Themen: den Unterschieden zwischen Wortschatzeinheiten und (mehr oder weniger) festen syntaktischen Konstruktionen und der Erweiterung des Wortschatzes durch ,Neologismen;, also ohne Recyclen bereits vorhandener Elemente. Im Vordergrund stehen aber die beiden zentralen Verfahren der Wortbildung, nämlich Derivation und Komposition, sowie der Ausblick auf konkurrierende Verfahren in anderen europäischen Sprachen.

Im Prinzip gibt es in unseren Sprachen zwei Bezeichnungsstrategien: eine syntaktische und eine lexikalische. Das Deutsche hat eine starke Tendenz zur lexikalischen Strategie. Das Kriterium der Festigkeit unterscheidet nicht konsequent zwischen beiden: Es gibt auch feste Syntagmen wie Paarformeln, nominale und verbale Phraseme bis hin zu Sprichwörtern. Die systematischen Unterschiede zwischen Phrasem und komplexem Wort sind aber relativ klar: Verteilbarkeit im Satz versus unmittelbares Aufeinanderfolgen, auf die Wortformen des Phrasems distribuierte Morphologie versus Fehlen von Binnenmorphologie, Vorkommen von Funktionswörtern versus Fehlen dieser Wörter.

Wenn der Wortschatz zumindest über die Zeit hin flexibel ist, sind Strategien der Erweiterung nötig. Von denen, die den Bestand nicht innovativ neu be- 
arbeiten, ist die Entlehnung am bedeutendsten. Anglizismen spielen, auch sprachübergreifend, eine wichtige Rolle, werden aber tendenziell überschätzt, sowohl in ihrer quantitativen Bedeutung als auch in ihrer „Fremdartigkeit“: Sie werden hybrid in den angestammten Wortschatz gemischt und auf vielen Ebenen assimiliert.

In der Wortbildung gibt es zwei dominante Verfahren: Derivation („Auseinem-mach-viele“) versus Komposition („Aus-zwei-mach-eins“). Beides sind weite Felder, die im Deutschen auch intensiv genutzt werden. Derivation ist bei den Inhaltswortarten (Substantiv, Adjektiv, Verb, teilweise auch Adverb) in allen von uns betrachteten Sprachen produktiv, meist in Form der Prä- und der Suffigierung; nur im Ungarischen finden sich nahezu ausschließlich Suffixe. Sprachübergreifend sind Affixe volatil, lassen sich also nicht auf eine Funktion eingrenzen; auch hier ist das Ungarische eine partielle Ausnahme. Romanische Sprachen bedienen sich primär des lateinischen Erbes an Affixen (wie auch an Stämmen), Englisch ist zwiegespalten; auch andere Sprachen partizipieren in ihrer Wortbildung an dem (gräko-)lateinischen Erbe. Die Komposition folgt einer ähnlich einfachen, aber rekursiven quasi-syntaktischen Regel wie die Derivation, wobei es zu strukturellen Ambiguitäten kommen kann. Das Deutsche ist besonders kompositionsfreudig; dafür sind vor allem die nominalen Determinativkomposita verantwortlich. Damit ähnelt das Deutsche Ungarisch und Englisch. Im Englischen gibt es jedoch eine unscharfe Grenze zu syntaktischer Attribuierung. Romanische und slawische Sprachen sind in der Komposition weniger produktiv, die romanischen Sprachen zeigen in der Regel linksköpfige Struktur und exozentrische Typen. Die deutsche Partikelverbbildung kann man als missing link zwischen Syntax und Wortbildung betrachten; es liegen gewisse Parallelen $\mathrm{zu}$ englischen und ungarischen verbalen Komplexbildungen vor; diese tendieren aber stärker zu syntaktischer Konstruktion als die deutschen.

Was im Deutschen durch (vor allem nominale) Begriffsbildung auf der Wortebene vollzogen wird, geschieht in anderen Sprachen oft durch unterschiedliche Attribuierungsverfahren (oder auch ganz anders: z. B. durch Ableitung). Insbesondere semantisch zu Determinativkomposition analoge Begriffsbildung nach dem Oberbegriff-Unterbegriff-Schema (bzw. gemäß Genus proximum und Differentia specifica) kann etwa statt durch Komposition durch syntaktische Komplexbildung erfolgen: Die „Differentia“ wird ausgedrückt durch Relationsadjektive, Possessivattribute, Präpositionalattribute, wobei oft - entsprechend dem nicht-referenziellen Status - um den Artikel reduzierte Formen gebraucht werden. Das Deutsche kennt marginal auch das, allerdings ist beim Genitivattribut keine Artikelreduktion möglich. 


\section{Zweite Runde: Rechtfertigen die vorgefundenen Charakterzüge eine Typisierung des Deutschen? Und wenn ja, welche?}

\subsection{Das Deutsche gemäß den hergebrachten Typologien}

Gehen wir zunächst von folgender Standardannahme aus: Das Deutsche ist wie andere indoeuropäische Sprachen ursprünglich fusionierend. Auf dem europäischen Kontinent ist daneben mit den finno-ugrischen Sprachen (dem Ungarischen, Finnischen und Estnischen) sowie dem Türkischen vor allem auch der agglutinierende Sprachtyp vertreten.

Bereits in Kapitel 4 wurde der Unterschied zwischen den beiden Sprachtypen an der Kasus-Numerus-Bildung des Substantivs erläutert. Hier sei noch eine kurze Rekapitulation eingefügt: In agglutinierenden Sprachen besteht bei den morphologischen Markern eine eineindeutige Zuordnung von Ausdruck und Inhalt. Jeder Marker realisiert genau eine Kategorie (nicht etwa mehrere gleichzeitig); nennen wir das ,separative Exponenz‘. Und: Jede Kategorie hat einen (nicht etwa mehrere) Exponenten; nennen wir das ,non-variante Exponenz‘. Für fusionierende Sprachen gilt die Negation beider Eigenschaften; wir haben es mit kumulativer und varianter Exponenz zu tun. Beispiele werden folgen.

Bei der Rückbesinnung auf den Urzustand allerdings müssen wir im Auge behalten, dass wir ihn und die weitere Vorgeschichte dieser Sprachgruppen - mangels Überlieferung - nicht kennen, sondern allenfalls schlüssige oder begründete Spekulationen anstellen können. Eine berühmte Spekulation geht dahin, dass die beiden Sprachtypen und ein weiterer, der isolierende, einander im Laufe der jeweiligen Sprachgeschichte ablösen, und zwar in einer Art Spirale. Die Idee eines solchen „Spirallaufs“ geht auf Georg von der Gabelentz, einen Sinologen und Sprachtypologen des ausgehenden 19. Jahrhunderts, zurück. Im Anschluss an Vladimir Skalička, bedeutender Typologe der Prager Schule, lässt er sich so skizzieren: ${ }^{1}$

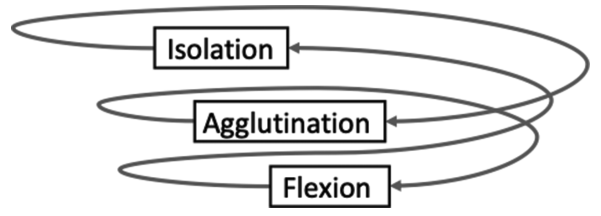

Abb. 18: Spirallauf der Sprachentwicklung.

Am Anfang der Spirale stehe das isolierende Muster: Lexikalische Wörter werden ohne Flexionsmarker aneinandergereiht; ihre Relation beruht wesentlich auf dem 
Mittel der linearen Ordnung. Selbstständige Wörter können gegebenenfalls für grammatische Information stehen wie etwa ein Quantifikator im Sinne von ,einige‘, ,mehrere‘, der in der isolierenden Sprache Burmesisch für die Markierung von ,Vielheit' steht, also semantisch in etwa der Pluralmarkierung entspricht. ${ }^{2}$ Aus solchen grammatischen Wörtern entwickelten sich dann unselbstständige, an die Inhaltswörter angeheftete Marker und damit einhergehend ein agglutinierender Sprachtyp. Durch die Vereinigung mehrerer Marker für die verschiedenen grammatischen Kategorien, ihre Vermengung und Reduktion zu einem kumulativen Marker entwickle sich daraus der fusionierende - früher auch ,flektierend“ genannte - Sprachtyp. Der anschließende Abbau der Flexionsmarker führe wieder zurück zum isolierenden Sprachbau. Jedoch nicht an den Ausgangspunkt zurück, sondern in einen veränderten Zustand. Danach beginne - allerdings von diesem anderen Startpunkt aus - ein erneuter Spirallauf.

Diese Hypothese bestätige sich - so Skalička und die ältere Forschung was die letzte Phase angehe, grosso modo für die westlichen (zumal die romanischen und germanischen) indoeuropäischen Sprachen; sie entwickelten sich vor allem im Bereich der nominalen Morphologie in unterschiedlichem Tempo vom fusionierenden zum isolierenden Typ. Die östlichen indoeuropäischen Sprachen, etwa das Armenische oder iranische Sprachen, hingegen gingen eher in Richtung Agglutination. Auch das Tschechische und andere slawische Sprachen teilten eher diese Tendenz zu agglutinierendem Umbau.

Aber ist dies nicht insgesamt, schauen wir auf unsere Sprachen, eine recht grobe Vereinfachung?

Da bestehe zum einen, so argumentiert z. B. Frans Plank, die Abgrenzung zwischen Agglutination und Fusion nicht nur auf zwei, sondern auf einer ganzen Reihe von Merkmalen - er nennt insgesamt elf solche Merkmale -, die voneinander logisch unabhängig seien und nicht unbedingt kovariieren müssen. ${ }^{3}$ So werde in den germanischen Sprachen, zumal im Deutschen, das Tempus Präteritum einerseits separativ, also unvermischt mit anderen Kategorien markiert. Andererseits aber habe es zwei verschiedene Ausdrucksformen: Ablaut und Dentalsuffix. Es bestehe also eine Art Mesalliance zwischen separativer und varianter Exponenz, oder anders gesagt: Ein Merkmal der Agglutination treffe auf ein Merkmal der Fusion. Zudem gibt es in der Deklination des deutschen Substantivs im Singular noch flexionsklassenabhängig eine Art reduzierte Fusion von Kasus und Numerus, so beim s-Suffix für Genitiv Singular der starken Maskulina und Neutra. Im Plural der Substantive hingegen liegt keine Kumulation mit der Kasusmarkierung vor, aber auch die Pluralmarkierung bedient sich verschiedener Marker. Wiederum stehen also ein Merkmal der Agglutination, die separative Exponenz, und ein Merkmal der Fusion, die variante Exponenz, im Widerspruch. Zu allem Überfluss kommt mit dem -(e)n des Dativ Plural (wie in Kind-er-n) noch ein 
agglutinierender Marker hinzu. Und wenn wir schon bei -(e)n oder Schwa (+ Konsonant) generell sind: Es handelt sich um ausgesprochen polyfunktionale Marker, die nicht nur in der nominalen, sondern auch in der verbalen Morphologie auftreten. Dies spricht immerhin für Fusion, da in agglutinierenden Sprachen die Marker nicht nur separativ und non-variant, sondern auch jeweils distinkt voneinander sind.

Quer zu der Unterscheidung zwischen Fusion und Agglutination (und weiteren morphologischen Typen) steht die zwischen Synthese und Analytizität. Diese, die auf den amerikanischen Strukturalisten Edward Sapir zurückgeht, war von Anfang an als eine graduelle Unterscheidung konzipiert. Man kann daher von einem in Zahlen ausdrückbaren Synthesegrad bzw. -index sprechen und meint damit die Anzahl der Inhaltseinheiten pro Wortform. So könnte man bei der Wortform gab von vier Inhaltseinheiten ausgehen: geb‘: + Präteritum + Singular +1 . Person/3. Person. ${ }^{4}$ Aber warum werden die Kategorien Singular und 1. Person/3. Person gezählt, obwohl keine entsprechenden Morpheme da sind, die Kategorie Indikativ aber nicht, die ja auch eine implizit kodierte Kategorie ist?

Insgesamt ist die Zählerei hochgradig problematisch, wenn auch tendenziell in die richtige Richtung weisend: Bei analytischer Realisierungsform wird das Bündel von Inhaltseinheiten, das in einer synthetischen Wortform vereinigt ist (oder sein könnte), auf mehrere Wortformen verteilt. Man denke an die Perfektform hat gegeben, wo die lexikalische Inhaltseinheit ,geb“ in einer anderen Wortform erscheint als die grammatischen Kategorien für Tempus, Person und Numerus. Sowohl fusionierende als auch agglutinierende Sprachen können einen hohen Synthesegrad haben: Hier stehen sich etwa das hochsynthetische fusionierende Polnisch und das hochsynthetische agglutinierende Ungarisch gegenüber. Die anderen indoeuropäischen Sprachen, die wir im Blick haben, sind insgesamt gesehen weniger fusionierend, aber nicht notwendigerweise weniger synthetisch. So findet sich synthetische (agglutinierende) Komparation auch im Englischen, das fusionierende Flexion weitgehend abgebaut hat.

Auch dem Deutschen wird ein Übergang vom synthetischen zum analytischen Sprachbau bescheinigt. Zu nennen sind etwa folgende Phänomene: ${ }^{5}$

1. Die beiden synthetischen Tempusformen des Germanischen werden im Laufe der deutschen Sprachgeschichte durch analytische Perfekt- und Futurformen ergänzt. Zudem wird das synthetische Präteritum teilweise durch das Präsensperfekt verdrängt.

2. Der synthetische Konjunktiv tritt in Konkurrenz zur analytischen würde-Form.

3. Im späten Mittelhochdeutschen bzw. Frühneuhochdeutschen kommen analytische Passivformen hinzu. 
4. An die Stelle von Kasus treten teilweise Präpositionen als analytische Realisierungsform bei der Verbkomplementation. Dies betrifft den Genitiv als Objektkasus besonders radikal. Im Attributbereich behauptet sich der Genitiv zumindest in der geschriebenen Standardsprache neben der Präposition von.

Allerdings: Es wird in all diesen Fällen durch die analytische Konstruktion nur ein Teil der zuvor synthetisch ausgedrückten grammatischen Kategorien realisiert, während ein anderer Teil, wie bereits für die analytischen Tempora angedeutet, nach wie vor synthetisch realisiert wird. Auch im nominalen Bereich (vgl. Punkt 4.) verliert der Kasus beim Ersatz durch eine Präposition zwar seine Funktion als Ausdruck der syntaktischen Relation; aber da Präpositionen ja Kasus regieren, bleibt Kasus auch in der Nominalphrase ein sich synthetisch manifestierendes Merkmal. Nur wird dies meist nicht am Kopfsubstantiv markiert, sondern am Determinativ oder einem adjektivischen Begleiter. Man kann daher von einem „diskontinuierenden (kombinatorischen) Verfahren“ zum Ausdruck von Kategorien(kombinationen) als Besonderheit des Deutschen sprechen. ${ }^{6}$ Dies als Erhöhung des Synthesegrades zu verbuchen ist jedoch etwas verkürzt. Eher haben wir es zu tun mit ,Analyse-cum-Synthese‘. Läge reine Analyse ohne jedes synthetische Beiwerk vor, so wären wir in der Tat bei einem isolierenden Sprachtyp. Dieser kann wohl, entgegen den Prognosen gemäß dem Spirallauf, realistischerweise kaum direkt von der Fusion aus erreicht werden, wie auch etwa das Englische zeigt. Es ist stärker analytisch und weniger fusionierend als das Deutsche, aber noch längst keine isolierende Sprache.

Gegen eine vorschnelle Einordnung in einen der bekannten Sprachtypen spricht letztlich auch, dass die Merkmale, z. B. pro und kontra ,fusionierend“ nicht in allen Bereichen des Sprachsystems gleichermaßen gegeben sein müssen. Im Französischen ist wie auch in anderen romanischen Sprachen die Nominalmorphologie weniger fusionierend und weniger synthetisch als die Verbalmorphologie. In dieser ist sogar noch in vielen romanischen Sprachen (nicht im Französischen) das synthetische Prinzip des Pro-Drop erhalten.

\subsection{Wettbewerbsmodelle: Wie kommt es zu Sprachentwicklung und Sprachverschiedenheit?}

Wie kommt die Vielfalt sprachlicher Ausdrucksformen in den Sprachen der Welt zustande, wie die Unterschiede zwischen „unseren“ Sprachen, wenn doch letztlich dieselben kommunikativen Ziele und daraus abgeleitet dieselben Sprachfunktionen realisiert werden sollen? Ich behandle diese Frage etwas ausführlicher, auch wenn dies das Porträt des Deutschen nur indirekt weiter ausleuchtet. 
Die moderne Antwort auf die Frage nach den Ursprüngen der Sprachverschiedenheit lautet so (oder zumindest so ähnlich): Unterschiedliche, miteinander im Wettstreit liegende Zielsetzungen führen zu dem divergierenden Befund, weil in einem Fall die eine Zielsetzung siegt, im anderen die andere. Die Idee eines Wettbewerbs ist, so scheint es, einer darwinistischen oder gar kapitalistischen Weltsicht geschuldet. Sie hat sich sogar in einem theoretischen Ansatz, der ,Optimalitätstheorie', niedergeschlagen. ${ }^{7}$ In diesem Ansatz spielen sehr abstrakte Prinzipien wie das der Ökonomie und das der Treue („faithfulness“) eine Rolle. Nach möglichst großer Ökonomie, möglichst geringem Formulierungsund Artikulationsaufwand strebe der Sprecher aus ureigenem Antrieb. Treue, also eine möglichst eindeutige Abbildung des zu Sagenden durch das Gesagte hingegen liege im Interesse des Hörers. Und da die Sprecher ja im eigenen Interesse auch die Interessen potenzieller Hörerinnen im Auge haben, liegen diese Ziele bei der Äußerungsgestaltung im Widerstreit.

Aber auch die funktional und historisch ausgerichtete Linguistik bedient sich dieses Modells. Sie spricht dabei von „Motivationen“ im Wettstreit. Dabei ist der Begriff Motivation hier problematisch, wie die Autoren eines einschlägigen Sammelbandes ${ }^{8}$ durchaus bemerken: Motivationen können nur Menschen haben, in diesem Fall also individuelle Sprecher oder auch miteinander kommunizierende Gruppen, nicht Sprachen. Die Verfechter und Verfechterinnen dieses Ansatzes gehen davon aus, dass die Sprecher ausschlaggebend sind für das Design „ihrer“ Grammatik, indem sie bei ihren Sprechakten eben aus einer Reihe möglicher Alternativen diejenige auswählen oder gar eine neue schaffen, die ihren jeweiligen „Motivationen“ am besten gerecht wird. So gebe es drei „externe“ Motivationen, die den Sprecher - in aller Regel unbewusst - bei der Produktion seiner Äußerung leiten: ${ }^{9}$ (1) das Bedürfnis, eine möglichst einfache und rasche Verarbeitung des Gesagten zu ermöglichen, also die Informationserschließung so einfach wie möglich zu gestalten, (2) das Bedürfnis, Form und Inhalt möglichst direkt oder gar ikonisch aufeinander zu beziehen und (3) das Bedürfnis, im Fluss der Rede möglichst nahe an der Dynamik des Diskurses bzw. der quasi natürlichen Form der Informationsvermittlung zu bleiben.

Bedürfnis (1) wird erfüllt, wenn wir z. B. das ,Gesetz der wachsenden Glieder befolgen. Es geht auf den Sprachwissenschaftler Otto Behaghel zurück, der beobachtete, dass bei Satzgliedern, deren Abfolge nicht absolut grammatisch determiniert ist, oft „das längere an zweiter Stelle“ stehe. ${ }^{10}$ So können wir zunächst einmal kompaktere Einheiten präsentieren, die bereits verarbeitet und abgespeichert werden können, bevor wir z. B. eine lange Phrase, die durch Nebensätze erweitert ist, folgen lassen. Oder indem wir, wie in Kapitel 5 (Abschnitt 3.2) gezeigt, einen Relativsatz getrennt von seinem Bezugswort in das Nachfeld verschieben. Oder auch: Wir stützen die Informationserschießung, indem wir die Träger kognitiv prominen- 
ter Information (wie der Agentivität oder Belebtheit) Trägern weniger prominenter Information vorangehen lassen. Bedürfnis (2) wird in den Sprachen, die wir überblicken, dadurch befriedigt, dass syntaktische Einheiten im Allgemeinen semantischen Einheiten entsprechen und nicht etwa mitten hinein z. B. in eine detailreiche Beschreibung des Agens in Form des Subjekts der Name des Patiens in Form des direkten Objekts „platzt“. Bedürfnis (3) hingegen wird dadurch Genüge getan, dass im Allgemeinen alte Information neuer Information vorausgeht - wir also nicht „mit der Tür ins Haus fallen“. Vielleicht imitieren wir damit auch den Fluss der Zeit von der bekannten Vergangenheit in die ungewisse Zukunft.

Wie aber entscheidet der Sprecher, wie löst er seine Zielkonflikte? Auch hier gebe es wieder drei - mit Metaphern aus der Sphäre der Politik oder gar des Kriegswesens umschriebene - Optionen: (i) friedliche Koexistenz, (ii) Teilen der Beute, and (iii) „der Gewinner bekommt alles“. ${ }^{11}$ Friedliche Koexistenz nach Strategie (i) sei dann möglich, wenn verschiedene Bedürfnisse durch dasselbe sprachliche Manöver befriedigt werden können. So erfülle die Subjekt-zuerst-Position im Englischen zugleich (nach Motivation (1)) das Bedürfnis, einer kognitiv prominenten, häufig agentiven Einheit den Vortritt zu lassen, die gleichzeitig in aller Regel auch alte oder bekannte Information repräsentiere und damit Motivation (3) genüge. ${ }^{12}$ Im Deutschen, so könnte man nun argumentieren, komme eher Strategie (iii) zum Zuge, wenn die erste Stelle im Satz, das Vorfeld, durch die Konstituente besetzt wird, die bekannte oder rahmensetzende Inhalte habe. In diesem Fall siege also Motivation (3). Ein Teilen der Beute (nach Strategie (ii)) kann man z. B. in folgender Konstruktion beobachten: Die Autorin ärgerte sich über die unfaire Kritik. Bei sich ärgern wie bei zahlreichen anderen deutschen reflexiven Verben der Gemütsbewegung gerät die kognitiv prominente belebte Informationseinheit in die syntaktisch prominente Subjektfunktion (vgl. Motivation (1)). Gleichzeitig kommt aber die Konstruktion auch Bedürfnis (2) entgegen, insofern als die Einheit, die den Auslöser der Emotion kodiert, in Form einer Phrase mit einer kausalen Präposition eine vergleichsweise explizitere und semantisch transparentere Kodierung genießt. Die konkurrierende Konstruktion wäre hier: Die unfaire Kritik ärgerte die Autorin.

Nun wissen wir ja allemal, dass die individuellen Entscheidungen der Sprecher nicht kreativ die jeweils optimale Grammatik schaffen oder aus dem Nichts emergieren lassen, sondern dass diese sich aus bestehenden Grammatiken bedienen. Der wesentliche Faktor für die individuelle Grammatik eines Sprechers ist die Konventionalität. Im günstigsten Fall bietet das erlernte und memorierte konventionelle System Alternativen für die Realisierung eines kommunikativen Ziels, zwischen denen der Sprecher je nach aktualer Motivationslage wählen kann. In beschränktem Maße hat er auch die Möglichkeit, eine mit den bestehenden Mitteln nicht erfüllbare Motivation durch Veränderung zu realisieren. So haben sich in der deutschen Sprachgeschichte ohne Zweifel zahlreiche Sprecher 
spontan und punktuell gegen aus ihrer Sicht oder eher ihrem intuitiven Gefühl überflüssige Komplikationen in der Flexionsmorphologie entschieden. Bestimmte starke Verben hatten im Präteritum nicht nur einen, sondern zwei Ablautvokale: er warf, aber sie wurfen. Transparenter und dienlicher für den Zusammenhang von Form und Funktion (vgl. (2)) ist es, wenn nur ein gegenüber dem Präsens ausgewechselter Vokal Präteritum signalisiert, nicht zwei. Analogie - das Prinzip ,Gestalte Formen nach dem Vorbild einer Form mit ähnlicher Funktion' - motivierte Sprecher und führte im Verein zum Wandel in Richtung ,Paradigmenuniformität‘. Auch der Verzicht auf die Genusdifferenzierung bei den Possessiva, also eine generelle Setzung von sein- für die 3. Person Singular, kommt dem Bedürfnis nach Einfachheit entgegen und wird schon einmal normwidrigerweise praktiziert. Vielleicht kann man sich einen realistischen Weg des Wandels so vorstellen: Er beginnt mit der vielfachen Wiederholung einer gelungenen Entscheidung im Sprachgebrauch eines Sprechers, die eine Art „Ansteckung“ der Interaktionspartner bewirkt. Diese wiederum zieht - im Sinne ,sozialer Selektion " - immer weitere Kreise, wird im Spracherwerb an die nächste Generation weitergegeben und führt schließlich zu Sprachwandel auf der Systemebene.

Auch den zentralen Auslöser für die allmähliche Ablösung eines rein fusionierenden Sprachtyps mag man letztlich dem Bedürfnis nach Ökonomie zuschreiben, genauer gesagt: einer „Maulfaulheit“ der Sprecher. Das Mittelhochdeutsche unterscheidet sich vom Althochdeutschen unter anderem dadurch, dass die Nebensilben „geschwächt“ wurden. Das heißt, in den Silben ohne Hauptakzent wurden in der Regel die vormals vollen Vokale zu Schwa oder sie entfielen auch ganz. Im Althochdeutschen lauteten z. B. die Pluralformen von ,Zunge' noch zungûn (Nominativ, Akkusativ), zungôm/zungôn (Dativ) und zungôno (Genitiv). (^ kennzeichnet Vokallänge.) Bereits im Mittelhochdeutschen haben wir einheitlich zungen. Man sieht darin eine Langzeitfolge der Fixierung des im Indoeuropäischen noch nicht festen Akzents auf die Stammsilbe in den germanischen Sprachen. Den nun grundsätzlich nicht mehr akzentuierbaren Nebensilben (oft Präfixe und vor allem Suffixe) wurde weniger artikulatorischer Aufwand zuteil. Sie bekamen allenfalls noch den Reduktionsvokal Schwa ab, bei dem der Mund kaum geöffnet wird und die Zunge in Ruhestellung verharrt. Unbeabsichtigte Folge war ein Zusammenfallen vorher unterschiedlicher Flexive, was ihre Funktionalität auf längere Sicht behinderte und, wiederum unbeabsichtigt, zum Ausweichen auf andere Mittel, z. B. der analytischen oder agglutinierenden Art, „motivierte“.

Allerdings bleiben viele Rätsel: Wenn z. B., wie in Kapitel 3 (Abschnitt 2.5) erwähnt, zahlreiche starke Verben dem Druck der Analogie und der Vereinfachung folgten und „schwach“ wurden - wie ist zu erklären, dass ebenfalls eine ganze Reihe von schwachen Verben stark wurde? Und so „inkonsequent“ verhielten sich auch die Sprecher des Englischen und Niederländischen. ${ }^{13}$ 
Können also diese Prozesse insgesamt ein funktionierendes kollektives System begründet haben? Was kann es bedeuten, wenn die synchrone Grammatik einer Sprache in eingefrorener Form die Prozesse der Entscheidung zwischen widerstrebenden Motivationen widerspiegeln soll, die einzelne Personen in individuellen Situationen fällen? Eine schlüssige Antwort darauf liegt kaum auf der Hand. Immerhin kann der Hinweis nützlich sein, dass Sprachwandel eine hochkomplexe Integration von Prozessen ganz unterschiedlicher zeitlicher Rahmengebung erfordert: ${ }^{14}$ von der in Sekundenbruchteilen erfolgenden Entscheidung des Sprechers für ein Wort, die Gestaltung der „richtigen“ Wortform und einer passenden syntaktischen Konstruktion über die länger anhaltenden Orientierungen an Standards der Interaktion (wie z. B. Geboten der Verständlichkeit oder Informativität) bis zur möglicherweise Epochen bestimmenden Wirkungsmacht sozialer Ordnungen. Diese setzen z. B. je spezifische Systeme der Differenzierung und Hierarchisierung sozialer Gruppen voraus und bestimmen damit stark die sprachliche Kennzeichnung von Personen und insbesondere den Ausdruck interpersonaler Beziehungen in direkter Interaktion. Im Inventar der Anredeformen und den Regeln ihrer Verwendung können sich ja mehr oder weniger direkt soziale Unterscheidungen und deren zeitliche Dynamik widerspiegeln. Ein lehrreiches Beispiel ist hier die komplizierte Entwicklung der Anrede für einen einzelnen Adressaten bzw. eine einzelne Adressatin im Deutschen, die von einem ursprünglich zweigliedrigen System im Mittelhochdeutschen über drei- oder sogar viergliedrige Systeme im 17. und 18. Jahrhundert wieder zu einem zweigliedrigen System führte, nämlich den Anredeformen du und Sie. ${ }^{15}$

Die Rolle des Sozialen wird allerdings in solchen Wettbewerbsmodellen leicht unterschätzt. Dabei hängt nicht unbedingt das Entstehen, sehr wohl aber das Überleben spontaner Änderungen entscheidend an sozialen Bedingungen. Dabei spielen neben unbeabsichtigten sozialen Effekten, den so genannten ,Phänomenen der dritten Art', auch bewusste Eingriffe, also Sprachregelungen eine Rolle. So haben sich normativ gesinnte Grammatiker und Spracherzieher über Jahrhunderte hinweg eingemischt und über Präskriptionen und Sanktionen die Gestalt der heutigen Standardsprache mitbestimmt und möglicherweise in einigen Punkten sogar entscheidend gesteuert. Zum Beispiel gab es bereits seit dem 14. Jahrhundert (außer im Ostmitteldeutschen) starke Tendenzen zum Wegfall von Flexionsendungen am Substantiv, insbesondere die ,Apokope‘ von -e. Noch heute entfällt in vielen Dialekten, so auch in meinem Heimatdialekt, bei Substantiven mit standardsprachlichem e-Plural der Marker am Substantiv, wie etwa bei [die Weg] statt die Wege. Zuweilen wird auch, um den Plural gemäß der „Motivation“ Numerusprofilierung zu markieren, auf den Umlaut zurückgegriffen wie bei [die Dääg] (für die Tage). Normierenden Eingriffen in der Zeit des 
Absolutismus, also im 16. und 17. Jahrhundert ist der Erhalt des -e als Pluralmarker und als Dativ-Singular-Flexiv zu verdanken. Zwar wurde das von gelehrten Grammatikern angestoßen, aber der Erfolg verdankt sich dem „kollektiv evolutionären Verhalten“16 einer ganzen Kaste von Sprachprofessionellen, also Kanzleischreibern, Geistlichen, Schulmeistern, Korrektoren oder „Sprachfreunden“ in den Sprachgesellschaften.

Das Schicksal des e-Flexivs am Substantiv kann aber auch die Sehweise derjenigen stützen, die denken, das Sprachsystem lasse auf längere Sicht nur „gute“ Lösungen zu, was auch immer als gut für das System oder ,systemadäquat‘ gelten mag. In diesem Fall sehen wir, dass sich das -e als standardsprachlicher Pluralmarker bewährt hat, während seiner Existenz als Dativmarker trotz aller Bemühungen ein baldiges Ende droht.

Auch in der Verbflexion ist -e labil. Gern lassen wir es in der 1. Person Singular Präsens in der informellen gesprochenen oder digitalen Kommunikation entfallen wie in ich komm, ich hab, ich hol usw. Hier ist die truthfulness im Vergleich mit der Setzung von -e nicht herabgesetzt; es kann also kompromisslos dem Gebot der Ökonomie gefolgt werden. So verwundert es nicht, dass in den Mundarten, die ja keinem normativen Diktat unterliegen, nicht nur der Wegfall von Schwa, sondern insgesamt der Abbau von Kasusunterscheidungen am Substantiv und anderen Flexiven weiter fortgeschritten ist als in der konservativeren Standardsprache. Beispielsweise fallen in Ausprägungen des Schwäbischen die Person-Numerus-Flexive des Verbs im Plural vollständig zusammen; man vgl. mir machet, ihr machet, sie machet. ${ }^{17}$ Den Genitiv, der ja auch in der Standardsprache deutliche Defekte aufweist, gibt es im gesamten süddeutschen Dialektraum praktisch nicht.

$\mathrm{Zu}$ bedenken ist aber, dass Vereinfachungen oft nicht ohne Kosten verlaufen. Das zeigt sich im Sprachvergleich. Wenn wie im Englischen Kasusunterscheidungen nicht mehr für die Unterscheidung syntaktischer Funktionen zumal die zwischen direktem und indirektem Objekt - zur Verfügung stehen, müssen andere Mittel herhalten, neben dem Einsatz von Präpositionen auch die Reglementierung der Satzgliedfolge. Der Ballast der Kasusmorphologie, über den vor allem diejenigen seufzen, die das Deutsche (oder auch das Polnische und andere stark fusionierende Sprachen) als Zweitsprache mühsam erlernen, kann nicht einfach und folgenlos abgeworfen werden. ${ }^{18}$

Derzeit erleben wir auf einem anderen Feld, dem der geschlechtergerechten Sprache, den Prozess eines intentional herbeizuführenden Sprachwandels hautnah mit. Ob dieser Wandel sich durchsetzen wird, ist eine spannende Frage. Im Augenblick gilt er vielen als moralisch erwünscht, erscheint aber etwa mit dem undifferenzierten Gebrauch des Gendersterns und seiner sprechsprachlichen Umsetzung als Hiatus nicht systemgerecht. Diesmal sind es nicht die Schulmeister, sondern Teile 
des intellektuellen Milieus im Kulturbetrieb, an den Universitäten und in den Medien, die sich für den in diesem Fall vorwärts gewandten Sprachwandel einsetzen.

\subsection{Das Deutsche: Porträt in aller Kürze}

Tragen wir nun doch die wesentlichen charakteristischen Züge des Deutschen zusammen: Es hat sich in der Morphologie seine ererbte fusionierende Flexion teilweise erhalten, und das mit einem auffallend geringen Inventar an Mitteln. Im Wesentlichen geht es dabei um Verbindungen von manchmal auch optionalem - e (Schwa) und einem oder maximal zwei Konsonanten oder auch nur um -e selbst. Damit wird sowohl die Person-Numerus-Morphologie am Verb als auch die KasusGenus-Numerus-Morphologie bei den nominalen Wortarten und die Komparation des Adjektivs bestritten. Die dadurch gefährdete Eindeutigkeit (,faithfulness“) der kodierten Information wird teils durch Übergang von rein synthetischer zu analytischer Kodierung kompensiert, in geringerem Maße auch durch die Neuinterpretation fusionierender als agglutinierende Marker (wie beim -(e)n des Dativs Plural). Eine deutsche Besonderheit ist die flexivische Kooperation in der Nominalgruppe, bei der die Ökonomie der Mittel und eine vergleichsweise transparente Kodierung der intendierten Funktion gleichermaßen gewährleistet sind. Auch das „eigentlich“ dem introflexiven Sprachtyp zuzuordnende Merkmal des Vokalwechsels (Ablaut, Umlaut) ist noch erhalten, nicht nur in der Tempus-Modus-Morphologie der starken Verben, sondern auch in der Wortbildung, ohne allerdings noch wirklich produktiv zu sein.

Der Ausdruck syntaktischer Relationen beruht, was die Komplemente angeht, teils auf Kasusunterscheidungen, also einem synthetischen Verfahren, teils auf dem Gebrauch von „semantisch ausgebleichten“ Präpositionen, einem analytischen Verfahren. Supplemente auf Satzebene werden fast ausschließlich präpositional, nicht nominal, somit analytisch realisiert. Auch bei dem neben dem Adjektiv wichtigsten Attributtyp, dem Possessivattribut, steht eine synthetische Realisierungsform als Nominalphrase im Genitiv neben einer analytischen in Form der Präpositionalphrase mit von. Anders als etwa im Englischen spielt aber die lineare Ordnung für den Ausdruck syntaktischer Relationen keine Rolle: Komplemente werden nicht durch ihre relative Stellung unterschieden. Übergreifende Gestaltungsprinzipien, etwa die Nominalhierarchien haben im Deutschen einen deutlichen Reflex, insofern als die pronominale Flexion bei maskulinen Pronomina und Determinativen im Singular noch die volle Palette der vier unterschiedenen Kasus aufweist.

Auch in der Syntax, insbesondere im Hinblick auf die lineare Ordnung, herrscht eine Art wohlgeordnete Uneindeutigkeit. Das Deutsche verfügt, geht man von den 
gängigen Schemata in der Wortstellungstypologie aus, über zwei Grundmuster der Satzgliedstellung: SVO für den Hauptsatz, SOV für den Nebensatz. (Daneben kennt das Deutsche wie viele andere Sprachen die für die Satzmodusunterscheidung wichtige Möglichkeit der Spitzenstellung des Finitums.) Charakteristisch ist aber vor allem die Satzklammer mit dem ungewöhnlichen Auseinanderrücken der Teile „analytischer Verbformen“ an zwei verschiedene, gegebenenfalls weit voneinander getrennte Positionen. Dabei können auch auffallend lange Verbketten in der rechten Satzklammer entstehen. Auch genießt das Subjekt keineswegs uneingeschränkt die lineare Vorrangstellung, sondern muss mit anderen Satzgliedern konkurrieren. Im Mittelfeld muss ein Kompromiss zwischen rigideren grammatischen Prinzipien der linearen Folge und einer freieren Ordnung nach dem Gewicht der Information gefunden werden. Auch in der Nominalphrase können dem Kopf die Nicht-Köpfe, die Attribute, sowohl vorangehen als auch folgen. Anders als beim Verb als Kopf im Verhältnis zu den Objekten sind aber die Plätze jeweils fest vergeben: Vor dem substantivischen Kopf erscheinen neben dem marginalen pränominalen Genitiv die adjektivischen Attribute - so komplex ausgebaut sie auch sein mögen. Auf den Kopf folgen alle Attribute in Form von Nominal- und Präpositionalphrasen und die satzförmigen Attribute. Das mag partiell wieder dem behaghelschen Gesetz geschuldet sein, führt aber auch zu einer klaren Domänenaufspaltung zwischen den (meist) qualitativen Attributen im Vorbereich und den referenziellen Attributen im Nachbereich des Substantivs. ${ }^{19}$

\section{Dritte Runde: Welche Rolle spielt das Deutsche in einem europäischen Sprachbund?}

Das Deutsche scheint, will man einer einflussreichen Publikation ${ }^{20}$ Glauben schenken, neun charakteristische Merkmale des „Standard Average European“ (SAE) zu erfüllen und gehört somit zusammen mit dem Französischen zum innersten Kern dieses Sprachbundes: (1) Es hat sowohl einen definiten als auch einen indefiniten Artikel; (2) Relativsätze werden durch „echte“ Pronomina eingeleitet und nicht etwa durch unveränderliche Partikeln; (3) es verfügt über ein haben-Perfekt; (4) es hat ein periphrastisches Passiv mit einem Partizip Perfekt und einem Hilfsverb; (5) es verfügt über die Möglichkeit eines,externen Possessors` im Dativ wie in Die Mutter wäscht [dem Kind] [die Haare] vs. Die Mutter wäscht [die Haare [des Kindes]]; (6) negative Pronomina wie niemand werden nicht mit einer zusätzlichen Negation beim Verb verbunden (also: Niemand kommt *(nicht)); (7) die Vergleichsgröße in der Gleichsetzungskonstruktion wird durch ein Relativadverb angeschlossen (wie in Sie ist so groß wie er); (8) Personsuffixe am Verb sind reine Kongruenzmarker; 
daher kein Pro-Drop; (9) Reflexivum und Intensifikator haben keine identischen Formen (also: sich vs. selbst).

Wenn wir andere europäische Sprachen, vor allem unsere Kontrastsprachen, in den Blick nehmen, so haben sie, mit Ausnahme des Französischen, nicht alle diese Merkmale. Das Niederländische und romanische Sprachen (Italienisch, iberoromanische Sprachen) und Albanisch teilen nur acht der neun Merkmale. Englisch hat sieben: Es hat zumindest nach dieser Darstellung keinen externen Possessor, und Reflexivum und Intensifikator fallen partiell zusammen. Polnisch und Ungarisch teilen jeweils nur fünf SAE-Merkmale. Beide Sprachen haben die Merkmale (2), (4), (5) und (7). Polnisch außerdem (9), Ungarisch außerdem (1). Sie verfügen also beide nicht über ein haben-Perfekt (3), in beiden Sprachen werden negative Pronomina mit einer zusätzlichen verbalen Negation verknüpft (6); beide Sprachen sind ProDrop-Sprachen (8). Polnisch hat keinen grammatikalisierten Artikel (1); im Ungarischen sind Reflexivum und Intensifikator identisch (9).

Nun ist die Idee von Sprachbünden und insbesondere die Aussonderung von SAE nicht unumstritten. Einer der wesentlichen Kritikpunkte ist die an den Anfängen der SAE-Idee sehr deutliche, jetzt immer noch spürbare Einseitigkeit zugunsten der westeuropäischen Sprachen. Man könne geradezu von einem „Westeuropa zugeneigten Eurozentrismus“ sprechen. ${ }^{21}$ Dabei würden vor allem die balto-slawischen Sprachen zu wenig in ihren europäischen Eigenschaften gewürdigt, während die Gemeinsamkeiten der südosteuropäischen Sprachen des so genannten Balkan-Sprachbunds schon länger gesehen wurden. Ob es allerdings weiterbringt, wenn statt des einen in sich ausdifferenzierten SAE gleich vier Typen (SAE Typ West, SAE Typ Ost, SAE Typ Süd-Ost und SAE Typ Russisch) angesetzt werden, ist eher fraglich. Immerhin erscheint eines im Hinblick auf das Deutsche sehr plausibel: Das Deutsche ist in mancherlei Hinsicht eine Brücke zwischen West und Ost: Das betrifft zum einen die Beibehaltung von Kasusunterscheidungen und das generelle Überleben synthetischer und fusionierender Merkmale in zum Teil veränderter Form, aber auch die gegenüber etwa dem Englischen und Französischen flexiblere Satztopologie. Wer will, mag darin einen Spiegel der geopolitischen Konstellation sehen. 
